\title{
Breaking waves in deep water: measurements and modeling of energy dissipation
}

\author{
Fadia Ticona Rollano ${ }^{1}$ (D) Adam Brown ${ }^{2} \cdot$ Ashley Ellenson $^{1} \cdot$ H. Tuba Özkan-Haller ${ }^{1,3} \cdot$ Jim Thomson $^{2} \cdot$ \\ Merrick C. Haller ${ }^{1}$
}

Received: 1 October 2018 / Accepted: 19 August 2019 / Published online: 6 September 2019

(C) The Author(s) 2019

\begin{abstract}
In the presence of strong winds, ocean surface waves dissipate significant amounts of energy by breaking. Here, breaking rates and wave-following turbulent dissipation rate measurements are compared with numerical WAVEWATCH III estimates of bulk energy dissipation rate. At high winds, the measurements suggest that turbulent dissipation becomes saturated; however, the modeled bulk dissipation continues to increase as a cubic function of wind speed. Similarly, the mean square slope (i.e., the steepness) of the measured waves becomes saturated, while the modeled mean squared slope grows linearly with wind speed. Only a weak relation is observed between breaker fraction and wind speed, possibly because these metrics do not capture the scale (e.g., crest length) of the breakers. Finally, the model skill for basic parameters such as significant wave height is shown to be sensitive to the dissipation rate, indicating that the model skill may be compromised under energetic conditions.
\end{abstract}

Keywords Wave breaking · Energy dissipation · Turbulence $\cdot$ Prediction of wave dissipation $\cdot$ Spectral wave model

\section{Introduction}

Wave breaking is one of the most important energy transfer mechanisms on the ocean surface. When a wave breaks in deep water, its energy is dissipated into turbulent kinetic energy, bubble injection, heat transfer, sea spray, and sound (Lamarre and Melville 1991; Melville 1996; Carini et al. 2015; Zippel and Thomson 2015):

$S_{\mathrm{ds}}=D_{\mathrm{TKE}}+D_{\text {buoy }}+D_{\text {heat }}+D_{\text {spray }}+D_{\text {sound }}$

Dissipation due to turbulent kinetic energy $\left(D_{\mathrm{TKE}}\right)$ and work against buoyancy ( $\left.D_{\text {buoy }}\right)$ are typically the most important terms contributing to the total wave-breaking dissipation $\left(S_{\mathrm{ds}}\right) . D_{\mathrm{TKE}}$ values are commonly obtained by depth-integrating the profiles

Responsible Editor: Richard Signell

Fadia Ticona Rollano

fadia.ticona.r@gmail.com

1 School of Civil and Construction Engineering, Oregon State University, Corvallis, OR, USA

2 Applied Physics Laboratory, University of Washington, Seattle, WA, USA

3 College of Earth, Ocean, and Atmospheric Sciences, Oregon State University, Corvallis, OR, USA of turbulent kinetic energy rates, $\epsilon(z)$, measured using Doppler sonars near the sea surface. The buoyancy work done on bubbles, however, is difficult to measure and cannot be resolved by Doppler sonars. A simplistic approach is sometimes applied to estimate wave-breaking dissipation by assuming it is equal to TKE dissipation only and that all the other dissipation terms are small (e.g., Carini et al. 2015; Thomson et al. 2016). This simplified relation has been shown to be accurate under moderate conditions, but neglecting the additional dissipation terms may lead to a significant underprediction of wave dissipation in the presence of strong winds (i.e., $U_{10}>15 \mathrm{~m} / \mathrm{s}$ ).

The importance of $D_{\mathrm{TKE}}$ and $D_{\text {buoy }}$ relative to $S_{\mathrm{ds}}$ has been the subject of several studies. For example, in a laboratory experiment, Lamarre and Melville (1991) found that for deep-water breaking waves, the contribution of buoyancy to the energy dissipation budget is $30-50 \%$, while a laboratory experiment by Blenkinsopp and Chaplin (2007) and numerical simulations by Deike et al. (2016) find this ratio to be between 5 and $15 \%$. More recently, laboratory work by Deane et al. (2016) suggests that bubble clouds may lead to an upper limit of turbulent dissipation rates of $\epsilon=$ $O\left(10^{2}\right) \mathrm{m}^{2} \mathrm{~s}^{-3}$. In the field, Thomson et al. (2016) observed a saturation of TKE dissipation rates for high wind conditions up to $20 \mathrm{~m} / \mathrm{s}$, though maximum values were only $\epsilon=$ $10^{-2} \mathrm{~m}^{2} \mathrm{~s}^{-3}$. Similarly, the TKE dissipation rates reported in Sutherland and Melville (2015), which were measured in 
winds up to $18 \mathrm{~m} / \mathrm{s}$, do not exceed $\epsilon=10^{-2} \mathrm{~m}^{2} \mathrm{~s}^{-3}$. Setting aside the issue of the vertical distribution of turbulent dissipation for the moment, both of these field studies indicate a similar upper bound in the integrated turbulence $D_{T K E}$. Yet, commonly used theories suggest that the bulk dissipation $S_{\mathrm{ds}}$ should increase with increasing wind speeds, following the logic that wind input and wave energy dissipation are in quasi-equilibrium (Phillips 1985), and wind input increases with wind speed (Donelan et al. 2006). This suggests that the relative role of $D_{\mathrm{TKE}}$ and other processes (such as $D_{\text {buoy }}$ ) changes significantly as wind speed increases. The discrepancy between the in situ measurements and models can be partially explained by the occlusion of turbulent velocity measurements in bubble plumes, as well as limitations in the depths of the measurements; recent work has estimated that these effects bias the in situ dissipation estimates low by up to a factor of four in high winds (Derakhti et al., in prep).

Improving the performance of wave forecasts likely requires improving our physical understanding of energy dissipation during wave breaking. Forecasting wave models solve the spectral action balance equation and, simply put, estimate wave energy dissipation as the energy that balances the input energy from wind forcing. In addition, some formulations assume pre-defined spectral shape constraints (such as an $f^{-5}$ diagnostic tail at the high-frequency end of the spectrum), while others incorporate bulk estimates of breaking and swell dissipation. These bulk parameterizations of energy dissipation do not explicitly resolve the physical processes involved in dissipating wave energy given in Eq. (1), and as such may limit the accuracy of spectral predictions.

Here, we analyze independent estimates of bulk wavebreaking dissipation and dissipation due to turbulence, not only against each other but also as they relate to wind speed, wave slopes, individual breakers, and overall predictability. The focus of this work is on high wind conditions, though not extreme conditions such as hurricanes.

To obtain estimates of turbulent dissipation rate $D_{\mathrm{TKE}}$, a Surface Wave Instrument Float with Tracking (SWIFT) is utilized. A SWIFT estimates turbulent dissipation rates in the upper layer of the water column using acoustic Doppler profilers. It also measures the wave spectrum and collects surface images for visual confirmation of wave breaking. The SWIFT was deployed twice in the winter of 2017, in the presence of strong winds up to $23 \mathrm{~m} / \mathrm{s}$.

To estimate bulk dissipation $S_{\mathrm{ds}}$ in the wave field, we employ the third-generation numerical wave forecasting model WAVEWATCH III (henceforth WW3). This model is generally skilled in predicting wave height (e.g., Bi et al. 2015; Yang and Neary 2017; Ellenson and Özkan-Haller 2018), lending some confidence to the wave energy dissipation estimates produced by the model. Different physics packages can be applied in WW3 to model the relationship between wind input energy and bulk wave energy dissipation. These packages differ from each other in the way they consider the details of breaking dissipation and the ramifications on spectral shape. The packages have evolved over the course of several decades to include increasing levels of understanding of the dissipation mechanisms by wave breaking, including the effects of dissipation of short waves by long breaking waves, or the effects of favorable or adverse winds.

Details of field collection activities and hindcast simulation setup are described in the "Observations and simulations of interest" section. In the "Comparison of measured and modeled wave parameters" section, we assess the skill of the model in estimating bulk wave parameters as compared with the measurements. In the "Energy dissipation" section, we explore the relationship between measured turbulent dissipation rates and modeled wave dissipation rates. Energy spectra and breaking metrics, in connection with energy dissipation, are discussed in the "Discussion" section. The influence of energy dissipation on the estimation of bulk parameters is also discussed in this section. Finally, we outline our conclusions in the "Conclusions" section.

\section{Observations and simulations of interest}

\subsection{SWIFT measurements}

A Surface Wave Instrument Float with Tracking was deployed from a helicopter offshore from the Oregon coast on two occasions in the winter of 2017. SWIFTs are free-drifting buoys equipped with GPS and IMU sensors to measure wavefollowing motion, Doppler profilers (Nortek Aquadopp HR) to measure turbulent velocity profiles, and cameras to take pictures of the sea surface. Data are recorded in 9-min bursts, sampled at $25 \mathrm{~Hz}$ every $12 \mathrm{~min}$. The additional $3 \mathrm{~min}$ is used to average, process, and telemeter the data collected during the previous $9 \mathrm{~min}$. Images are captured every 4-6 s. An in-depth description of how SWIFTs operate is found in Thomson (2012).

Each of the deployments was at the onset of significant storm events that were carefully selected by monitoring wave forecasts for the Oregon coast. On both occasions, the SWIFT was released approximately $23 \mathrm{~km}$ due west of Newport, OR, and allowed to drift freely to shore. The trajectory taken by the SWIFT during each event is shown in Fig. 1. The first deployment was on February 16, 2017, and lasted about 4 days. After its release, the SWIFT floated northward towards the coast but began drifting westward between Neskowin and Pacific City as a result of offshore-directed currents. After making a loop that took the buoy almost as far south as Lincoln City, it continued to drift northward along the coast until it was recovered from the spit of Netarts Bay. In contrast, the second deployment was shorter, beginning on April 7, 2017, and lasting just over a day. After its release, the buoy followed a fairly straight path and was recovered just south of Cape Lookout. 

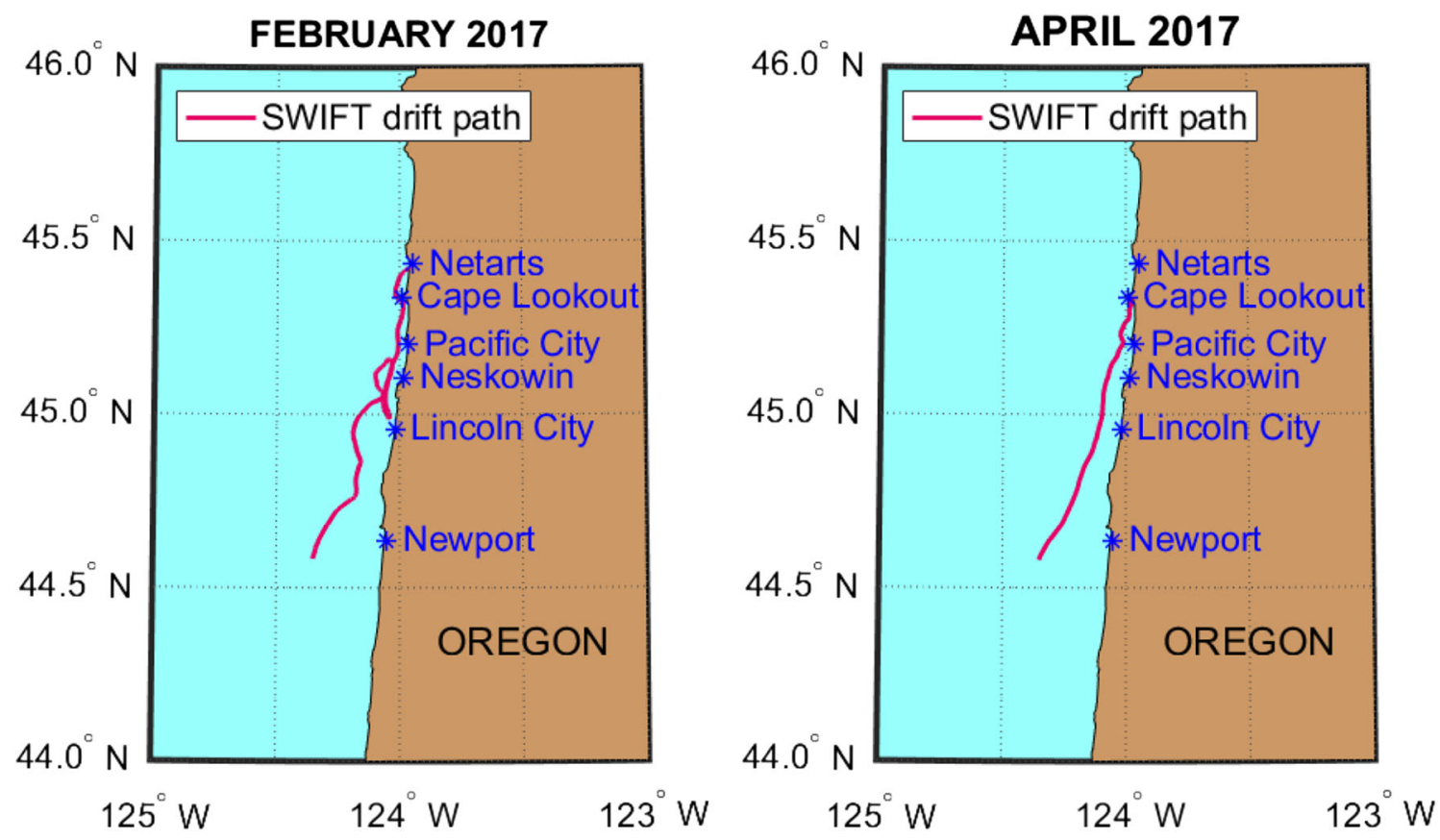

Fig. 1 SWIFT buoy drift paths during February and April 2017 deployments

To keep our scope on whitecapping - as opposed to nearshore, depth-limited, wave breaking - we only utilize observations made at depths greater than $50 \mathrm{~m}$ in our analysis. In both deployments, this cut-off point roughly corresponds to a non-dimensional $(\mathrm{kh})$ depth of 2.5 and allows us to focus on wave breaking in deep water and in upper intermediate-water. In addition, we observed that during the April 2017 event, when the SWIFT first reached a 50-m water depth, the wind speed had dropped to roughly $15 \%$ of that recorded at the beginning of the deployment (from 22.3 down to $3.7 \mathrm{~m} / \mathrm{s}$ ), giving us confidence that this threshold captures the observations of whitecapping driven by strong winds.

\subsection{WAVEWATCH III hindcasts}

The observed events were simulated using WW3 version 5.16. As stated in the user manual, WW3 solves the spectral action balance equation:

$\frac{\partial N}{\partial t}+\nabla \cdot \dot{x} N+\frac{\partial}{\partial k} \dot{k} N+\frac{\partial}{\partial \theta} \dot{\theta} N=\frac{S}{\sigma}$

where $N \equiv E(k, \theta, t, \boldsymbol{x}) / \sigma$ is the wave action. $E$ is the energy spectrum as a function of wavenumber $k$, direction $\theta$, time $t$, and two-dimensional space $\boldsymbol{x} . \sigma$ is the relative (radian) frequency moving with the mean current (The WAVEWATCH III Development Group 2016). Variables with a dot accent above denote time derivatives. On the right hand of Eq. (2), $S$ represents the net effect of sources and sinks to the energy spectrum, each of which is parameterized in the different physics packages available in WW3. In deep water, the most important source and sink terms are:

$S=S_{\mathrm{in}}+S_{\mathrm{ds}}+S_{\mathrm{nl}}$

where $S_{\text {in }}$ is the wind input term, $S_{\mathrm{ds}}$ the dissipation term, and $S_{\mathrm{nl}}$ represents non-linear wave-wave interactions. Additional terms may be added as needed to describe other processes such as bottom friction and depth-limited wave breaking in shallow water.

The most commonly used physics packages, implemented to describe the interrelation of $S_{\text {in }}$ and $S_{\mathrm{ds}}$, are ST2, ST4, and ST6. The widely used ST2 package, developed by Tolman and Chalikov (1996), uses different criteria for dissipating energy at low and high spectral frequencies; at low frequencies, the dissipation parameterization is akin to turbulent dissipation, and at high frequencies, an $f^{-5}$ spectral roll-off is prescribed. The formulation in the ST4 physics package, developed by Ardhuin et al. (2010), forgoes a pre-defined spectral shape and rather parameterizes energy dissipation based on steepness for swell conditions, and exceedance thresholds that capture directionality and dissipation of short waves by long breaking waves. The most recent physics package, ST6, was developed by Zieger et al. (2015) with a special focus on whitecapping dissipation. ST6 accounts for negative wind input (i.e., both favorable and adverse winds) and full air-flow separation and, after exceeding a steepness threshold, implements a dissipation formulation that depends on (1) an inherent breaking dissipation term as a function of the spectral density and (2) a forced term that becomes active at frequencies greater than the spectral peak, both proportional to the 
threshold exceedance. ST6 also enforces a spectral shape with an $f^{-5}$ spectral roll-off. For a complete description, the reader is referred to the references cited above and the WW3 user manual (The WAVEWATCH III Development Group 2016).

In this study, the ST6 physics was chosen because of the emphasis on whitecapping and wave turbulence in its formulation. The non-linear wave-wave interaction term $S_{\mathrm{nl}}$ was parameterized using the discrete interaction approximation (DIA) package described in Hasselmann et al. (1985).

The WW3 hindcasts were produced using three nested grids of increasing resolution as developed in a previous study of the Oregon coast completed by Ellenson and Özkan-Haller (2018). The largest of the grids covered the entire Pacific Ocean at a 30 arc-minute resolution. The second grid spanned the eastern North Pacific at a 7.5 arc-minute resolution. The third grid extended over the continental shelf $\left(40.3^{\circ}-49.5^{\circ}\right.$ $\mathrm{N}, 233^{\circ}-236.25^{\circ} \mathrm{W}$ ) at a 90 arc-seconds resolution.

The bathymetry for all grids was obtained from the National Geophysical Data Center's ETOPO1 (Amante and Eakins 2009), and the third grid was also integrated with bathymetry from NOAA's Gridded Tsunami Bathymetry. Wind input at 10-m elevation and air-sea temperature differences were obtained from NCEP's Global Forecast System (GFS), at a $0.5^{\circ} \times 0.5^{\circ}$ spatial resolution and 3-h time resolution. Wave frequencies are resolved between 0.003 and $1.23 \mathrm{~Hz}$ with a resolution ranging from $0.003 \mathrm{~Hz}$ for low frequencies up to $0.11 \mathrm{~Hz}$ for high frequencies. The wave directional resolution is $10^{\circ}$.

The output resolution of the hindcasts is $1 \mathrm{~h}$. The output of the directional wave spectra was obtained at pre-determined spatial points chosen to match the mean location of the SWIFT at each hour of each deployment. In addition, several other parameters were computed over a grid spanning the finest resolution grid described earlier. Standard mean wave parameters that are available on a grid include significant wave height, mean wave period, and peak wave direction. Gridded parameters of the atmosphere-waves layer include wind-to-wave energy flux and friction velocity. The gridded output of wave to sea energy flux (i.e., depth-averaged wave dissipation rate) was also obtained.
Figure 2 shows a time series of wind speed from the GFS at the spatially varying SWIFT locations for each deployment. The highest wind speed during the February 2017 event was $16.5 \mathrm{~m} / \mathrm{s}$ at the beginning of the deployment. After $14 \mathrm{~h}$, the wind speed dropped to a local minimum of $3.0 \mathrm{~m} / \mathrm{s}$ and then it oscillated between that value and $9.5 \mathrm{~m} / \mathrm{s}$ until the SWIFT arrived at the coast. For the April 2017 deployment, initial winds were a maximum of $22.3 \mathrm{~m} / \mathrm{s}$. As the deployment progressed the wind speed generally decreased, reaching a local minimum of $3.5 \mathrm{~m} / \mathrm{s}$ after $25 \mathrm{~h}$.

\subsection{Observed and modeled conditions}

Sample gridded and spectral WW3 output of the first hour of each deployment is shown in Fig. 3. An image of a whitecap taken from the SWIFT closest to the indicated time is also shown in the figure. On the heat maps of significant wave height and dissipation, the areas with the largest values are linked to the presence of storms in both events, with the wave conditions during the April storm being the most energetic. This is corroborated by looking at the sample images in the figure; the one taken during the second event shows the most foam.

At the first hour of the February event, waves were predominantly coming from the SW, with a significant wave height of $6.3 \mathrm{~m}$ and a mean period of $9.8 \mathrm{~s}$. Later during the deployment (not shown), the largest area of high significant wave height and dissipation shifted northward and away from the buoy. The smaller energetic area south of the SWIFT remained roughly in place and faded over time. At the first hour of the April event, mean waves were coming from the SSW, with a significant wave height of $7.3 \mathrm{~m}$ and a mean period of $9.5 \mathrm{~s}$. In later hours of this deployment (not shown), the storm-associated area on the grid plots shifted northward along the shelf as the SWIFT drifted towards the coast. Overall, as both deployments progressed, the wave conditions became less energetic and fewer waves were captured breaking by the SWIFT camera.
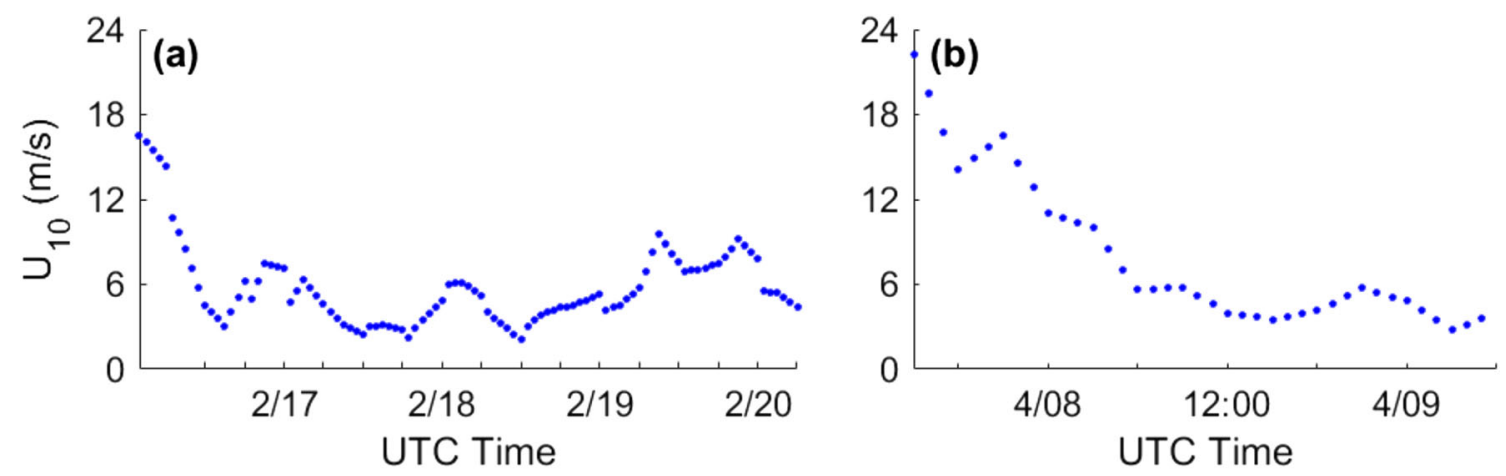

Fig. 2 Time series of wind speed at $10 \mathrm{~m}$ above the water surface elevation at the SWIFT location corresponding to the a February 2017 and $\mathbf{b}$ April 2017 deployments 
Fig. 3 WW3 gridded output of significant wave height $\left(H_{\mathrm{s}}\right)$ and wave to sea flux $\left(S_{\mathrm{ds}}\right)$, including wave conditions at the SWIFT (in text); WW3 energy spectra at SWIFT location; and sample pictures of the sea surface taken from the SWIFT during the first hour of the a February 2017 and b April 2017 deployments
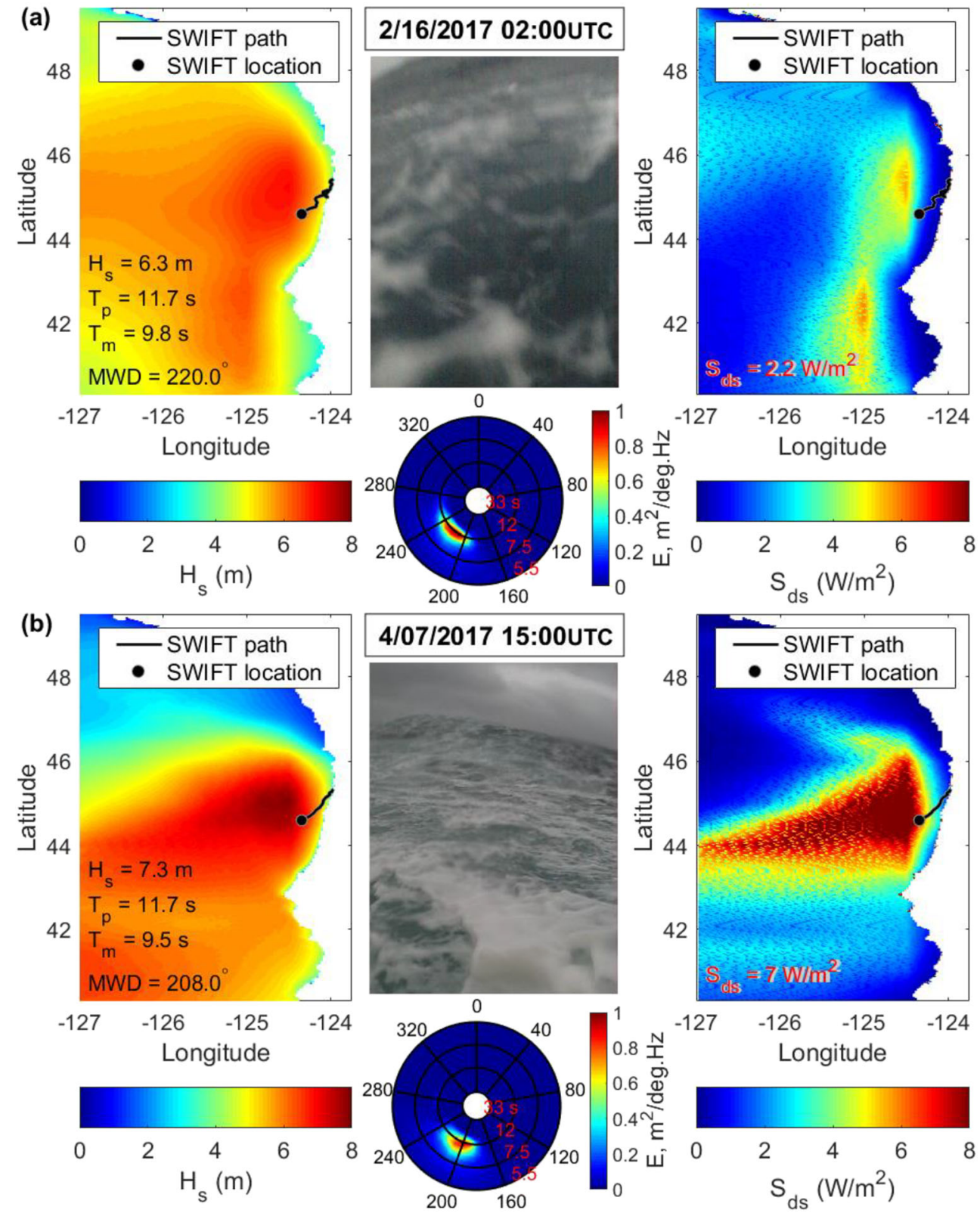

\section{Results}

\subsection{Comparison of measured and modeled wave parameters}

All WW3 gridded parameters were spatially interpolated to obtain SWIFT-following time series to enable model/data comparisons. The interpolation was done bilinearly by first finding the grid quadrant that contained the location of the point of interest that is the mean SWIFT position at a given hour. WW3 output values at the SWIFT locations were then found by linearly interpolating in one direction (west-east) and then the other (north-south).
The time series of bulk parameters measured with the SWIFT and computed with WW3 are shown in Fig. 4. Model performance metrics were calculated following formulas presented in García-Medina et al. (2014). The $R^{2}$ values for significant wave height during the February and April 2017 events are 0.94 and 0.95 , respectively. Lower skill is shown in simulating the mean wave period, with a general negative bias by the model. For the February 2017 deployment, the estimates of peak wave direction (PWD) have a skill of $R^{2}=0.39$. February PWD errors are more pronounced for times when the SWIFT buoy followed a looped drift path south of Cape Lookout due to the aforementioned offshore-directed currents. Meanwhile, for the second deployment, the modeled estimates of PWD have an $R^{2}$ value of 0.93 . 
Fig. 4 Time series of measured and modeled bulk parameters at mean hourly SWIFT locations for the February 2017 (left column) and April 2017 (right column) deployments. a, b Significant wave height. c, d Mean wave period. e, f Peak wave direction
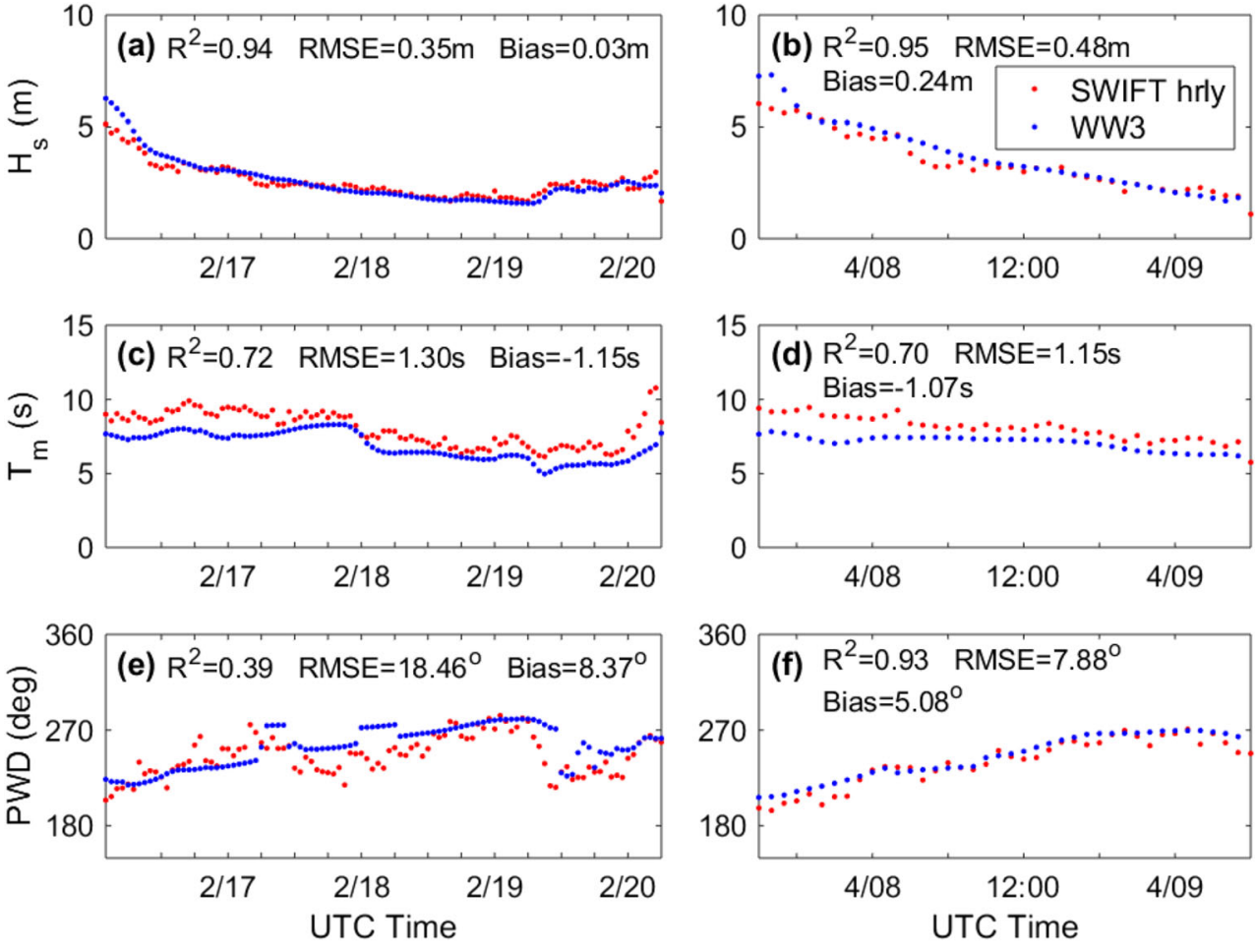

\subsection{Energy dissipation}

Doppler sonars on board the SWIFT were used to measure turbulent velocity fluctuations and estimate turbulent kinetic energy rate profiles, $\epsilon(z)$, over the top $0.62 \mathrm{~m}$ of the ocean immediately below the instantaneous sea surface. The methods apply the second-order structure function, with details described in Thomson (2012). Prior to calculating the structure function, the raw velocity data are screened to remove points with low correlations. These are known to be associated with high void fractions and bubble clouds in the water column. Thus, the observed dissipation rates preferentially represent the persistent turbulence related to wave breaking outside of episodic bubble injection events. The recent work of Derakhti et al. (in prep) suggests that this screening of the raw data results in dissipation estimates that are biased low by up to a factor of 3. Combined with the finite depth of the measurements, Derakhti et al. (in prep) indicate that the overall bias in high sea states is a factor of 4 .

The calculations are carried out for each 512-s burst of SWIFT data, and the result is a burst-averaged dissipation rate profile, $\epsilon(z)$, every $12 \mathrm{~min}$. For a discussion of dissipation rates calculated on a wave-by-wave basis, see Thomson et al. (2016). To evaluate our dataset, we test the depth dependence of dissipation rate following Terray et al. (1996). The following proportionality, derived by way of non-dimensional scaling, governs the wave turbulence layer:

$$
\left(\frac{\epsilon(z) H_{s}}{F}\right) \propto\left(\frac{z}{H_{s}}\right)^{-\lambda}
$$

Here, $\epsilon(z)$ is the TKE dissipation rate measured at depth $z$, $H_{s}$ is the significant wave height, $F$ is the wind-to-wave input energy rate, and $\lambda$ is a tuning parameter. For the combined data from both deployments, this dependence is best fit by a tuning parameter of $\lambda_{\text {combined }}=1.3$, matching previously reported fits using analogous wave-following data. In Gemmrich (2010), the tuning parameter reaches upward values of $\lambda_{G 10}=1.6$ when breaking is confirmed $\left(\lambda_{G 10}=1.1\right.$ when wave conditions are calm), Sutherland and Melville (2015) reported values of $\lambda_{S M 15}=1$ close to the sea surface and $\lambda_{S M 15}=2$ at depths greater than one significant wave height, and in Thomson et al. (2016), the depth dependence is characterized by $\lambda_{T 16}=1.4$ for wind observations up to $20 \mathrm{~m} / \mathrm{s}$.

The profiles of TKE rates measured by the SWIFT are shown in Fig. 5. Each line represents the averaged dissipation rate during a 12-min burst and is colored by breaker fraction, $Q_{b}$, for that burst. The method for calculating breaker fraction is described in the forthcoming "Consideration of individual breakers" subsection. The dashed black line in Fig. 5 is the background signal $\epsilon_{0}(z)$, a persistent base measurement of turbulence even in the absence of wave breaking. The background signal was estimated by averaging numerous profile observations during periods when no visible breaking activity was recorded.

The color information shown in Fig. 5 illustrates the correlation between high values of breaker fraction and most profiles of high dissipation rates. The light gray lines are profiles 
Fig. 5 Observed near-surface TKE profiles $\epsilon(z)$ and background signal $\epsilon_{0}(z)$ for the a February 2017 and b April 2017 deployments

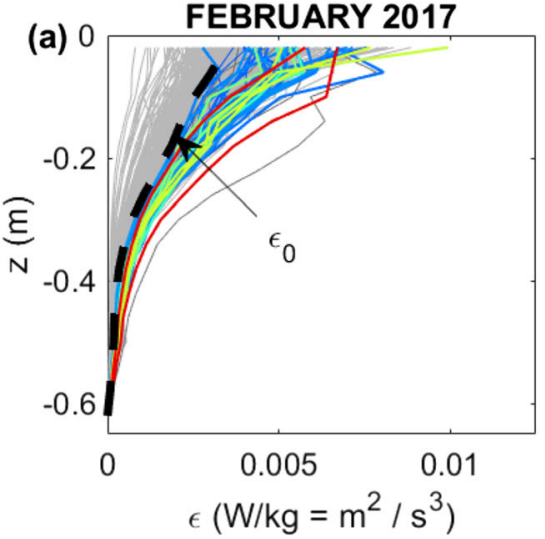

that have a breaker fraction equal to or lower than 0.02 . All the lines in color have dissipation values greater than the background signal at all, or at least most, depths. Near the surface, the value of the greatest dissipation rate was recorded during the April 2017 deployment $\left(\epsilon_{z}=-0.02 \mathrm{~m}=0.012 \mathrm{~m}^{2} / \mathrm{s}^{3}\right)$ with a corresponding breaker fraction of 0.04 . The highest breaker fraction estimated was also for the April 2017 deployment, with a value of 0.07 and a corresponding dissipation rate of $0.005 \mathrm{~m}^{2} / \mathrm{s}^{3}$ near the surface.

Next, we express the measured turbulent dissipation rate in units of energy rate per unit area to match those of WW3 wave-breaking dissipation $\left(S_{\mathrm{ds}}\right)$. To do so, the near-surface TKE profiles, with the background signal removed, are integrated over depth and multiplied by the density of water. $D_{\mathrm{TKE}}$ in $\mathrm{Eq}(5)$ is then the excess depth-integrated turbulent kinetic energy dissipation rate observed by the SWIFT.

$D_{\mathrm{TKE}}=\rho_{\text {water }} \int \epsilon(z)-\epsilon_{0}(z) d z$

The logarithmic time series of SWIFT turbulent dissipation rates and WW3 wave dissipation rates are shown in Fig. 6. Generally, bulk wave dissipation from the model was greater than the turbulent dissipation observed in the field by no more than $0.2 \mathrm{~W} / \mathrm{m}^{2}$ under moderate wind conditions, but by up to $1.8 \mathrm{~W} / \mathrm{m}^{2}$ in February of 2017 and $6.3 \mathrm{~W} / \mathrm{m}^{2}$ in April of 2017 in the presence of strong winds when whitecapping was observed. The difference between $D_{\mathrm{TKE}}$ and $S_{\mathrm{ds}}$ under highly energetic conditions is likely due to the increased importance of other dissipation mechanisms besides turbulence, such as buoyancy due to bubbles in the water column, as well as bubble occlusion in the Doppler data (Derakhti et al., in prep). This is primarily the case at the beginning of both deployments when wind speed observations were the highest (see Fig. 2).

Although it has been argued that wind energy input rate closely balances the rate of energy dissipation by breaking in moderate conditions (Thomson et al. 2016), the functional form of the wind dependence remains debated in the literature, especially in high wind conditions. Figure 7 reveals distinct trends in the dissipation-wind relation for SWIFT and WW3 dissipation estimates. On average, SWIFT $D_{\text {TKE }}$ increases linearly with wind speed up to $11 \mathrm{~m} / \mathrm{s}$, but plateaus at about $0.5 \mathrm{~W} / \mathrm{m}^{2}$ for greater wind speeds. Meanwhile, WW3 $S_{\mathrm{ds}}$ increases with increasing wind speed following a cubic relationship. The latter is not entirely surprising since previous studies have shown that both energy input into the wave field as well as wave dissipation increase with wind speed such that wave dissipation is well approximated by the cube of the wind friction velocity (e.g., Craig and Banner 1994; Thomson et al. 2016). The apparent saturation of turbulent dissipation at high wind speeds, contrasted with the cubic trend between WW3 bulk dissipation and wind speed, is a key finding of this study. It suggests that other breaking dissipation mechanisms, such as bubble dynamics and sea spray, may become more
Fig. 6 Logarithmic time series of dissipation rates for the a February 2017 and b April 2017 deployments. Gray and red dots are observations of depthintegrated SWIFT turbulent dissipation rate $\left(D_{\mathrm{TKE}}\right)$ in 12-min and 1-h bins, respectively. Blue dots are hourly estimates of WW3 depth-averaged dissipation rate $\left(S_{\mathrm{ds}}\right)$
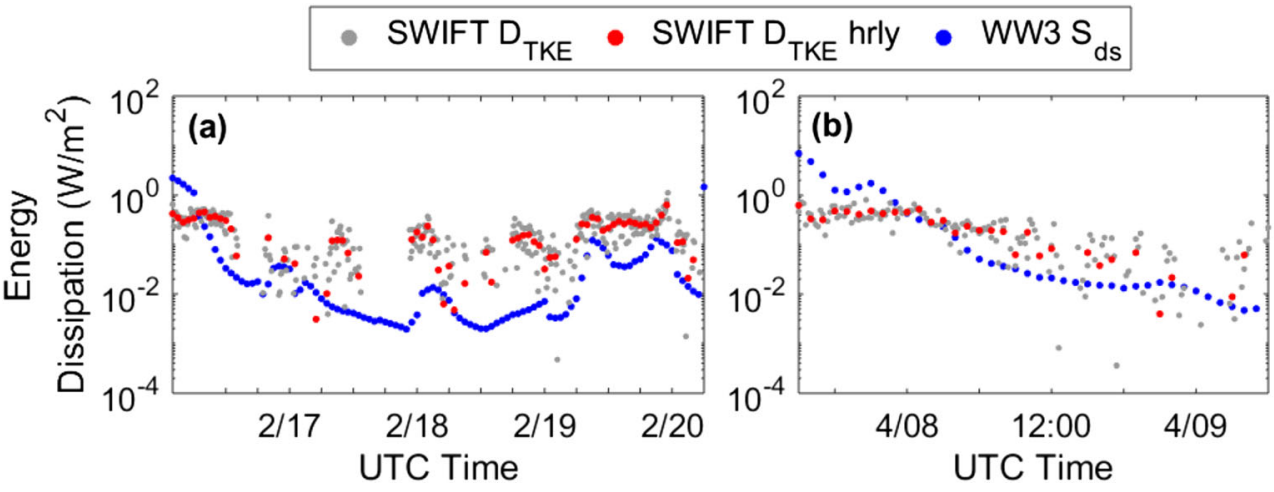
Fig. 7 SWIFT depth-integrated turbulent dissipation rate with background signal removed (top) and WW3 depth-averaged dissipation rate (bottom) versus $10-\mathrm{m}$ wind speed. Data points corresponding to the February 2017 deployment are shown in cyan and in orange for the April 2017 deployment. Filled circles indicate data points taken at a water depth greater than $50 \mathrm{~m}$
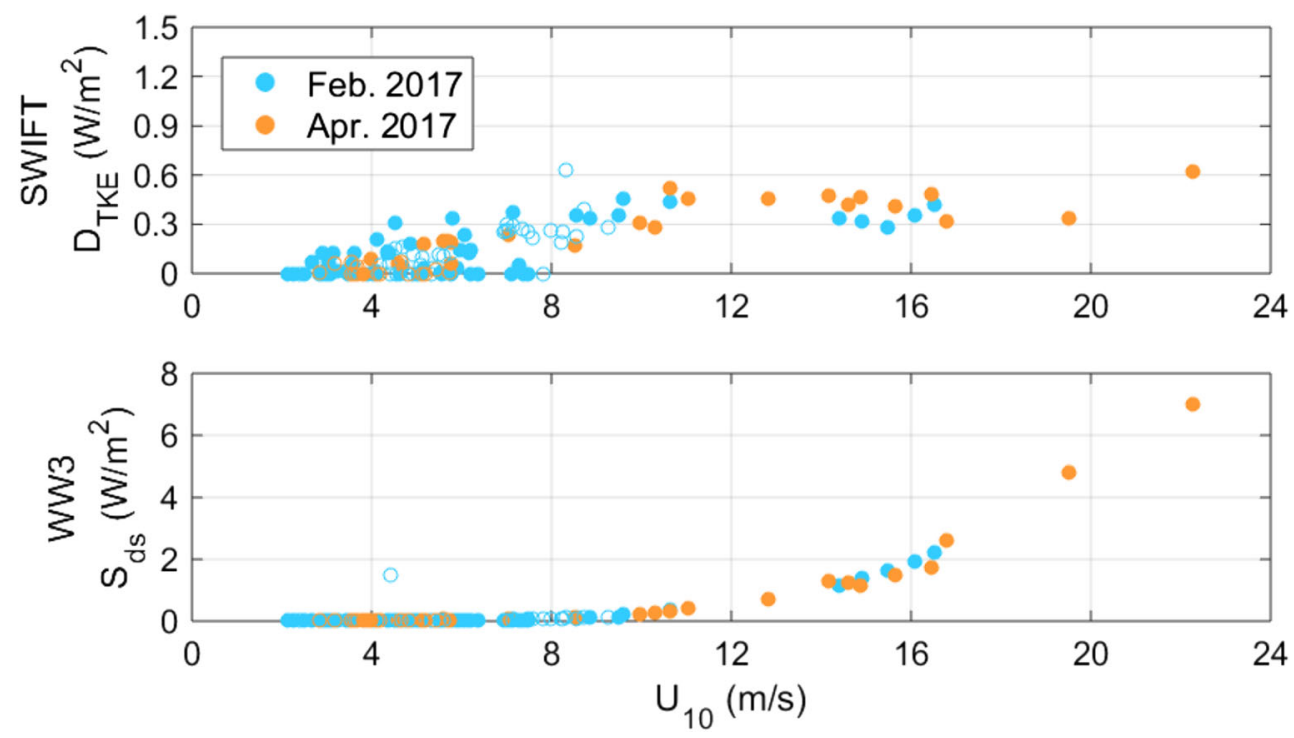

prevalent as the winds increase above $15 \mathrm{~m} / \mathrm{s}$. Another possible explanation may be the saturation of energy input from wind to waves, e.g., very high humidity at the air-sea interface that limits energy transfer even at high wind speeds. Alternatively, it is possible the burst-averaged dissipation rate values from the SWIFT estimates do not adequately capture the dissipation occurring during intermittent breaking, in part because the Doppler sonars do not work well in high void fractions. These measurement effects are expected to be small at low wind speeds and then grow to approximately a factor of four at high winds (Derakhti et al., in prep). Adjusting for this affect in Fig. 7 would cause the SWIFT dissipation estimates to continually increase with wind speeds; however, there would still be an imbalance with the WW3 model dissipation.

\section{Discussion}

In this section, we analyze wave spectra and breaking metrics, to explore the linkage between wave breaking, energy dissipation, and wind speed. We evaluate plausible mechanisms for the observed saturation of turbulent dissipation rates at high winds. We also asses the influence of energy dissipation on model performance.

\subsection{Influence of spectral shape}

Measured and modeled wave spectra, integrated over direction, are compared in Fig. 8. The model matches the observed unidirectional spectra the best when wind speeds are neither too high nor too low (middle column). When wind speeds are highest (left column), the high-frequency energy is slightly overpredicted by the model. On the other hand, when wind speeds are lowest (right column), the energy spectra are slightly underpredicted by the model at high frequencies. The spectra are best correlated at frequencies below the peak, while the most notable discrepancies occur at frequencies higher than $0.3 \mathrm{~Hz}$.

A significant parameter associated with wave breaking, which is derived from the wave spectra, is the mean squared slope (mss) (e.g., Schwendeman et al. 2014). This is related to wave steepness and is often better correlated with breaking than the bulk wave steepness $H_{s} k_{p}$. Following Banner et al. (2002), we first compute the spectral saturation:

$B(f)=\frac{(2 \pi)^{4} f^{5} E(f)}{2 g^{2}}$

Then, we obtain mss in the frequency range $f_{1}<f<f_{2}$ by applying the following equation:

$\mathrm{mss}=\int_{f_{1}}^{f_{2}} \frac{2 B(f)}{f} d f$

In this study, the mean squared slope is calculated from measured and modeled spectra over a frequency range of $0.045-0.49 \mathrm{~Hz}$, which is the range of frequencies resolved by the SWIFT instrumentation. This slope metric is effectively the fourth moment of the scalar energy spectrum, and thus, the higher frequencies (which show the significant model-data difference in Fig. 8) are most important.

Time series of mss corresponding to both 2017 events are shown in Fig. 9. Generally, there is good agreement between mss calculated from SWIFT and WW3 spectra, but there are greater discrepancies at the beginning of the deployments when mss (and wind speed) values are highest. In these high wind conditions, the WW3 values are notably higher than the measured values, just as with the dissipation rates. Whereas the SWIFT observations of mss are initially 0.013 and 0.014 for the February and April deployments, respectively, the WW3 corresponding estimates are 0.025 and 0.031 . 
Fig. 8 Measured and modeled frequency spectra at the SWIFT location at different times (UTC) and different $10-\mathrm{m}$ wind speeds. Top panels correspond to the February 2017 deployment and the bottom panels correspond to the April 2017 deployment
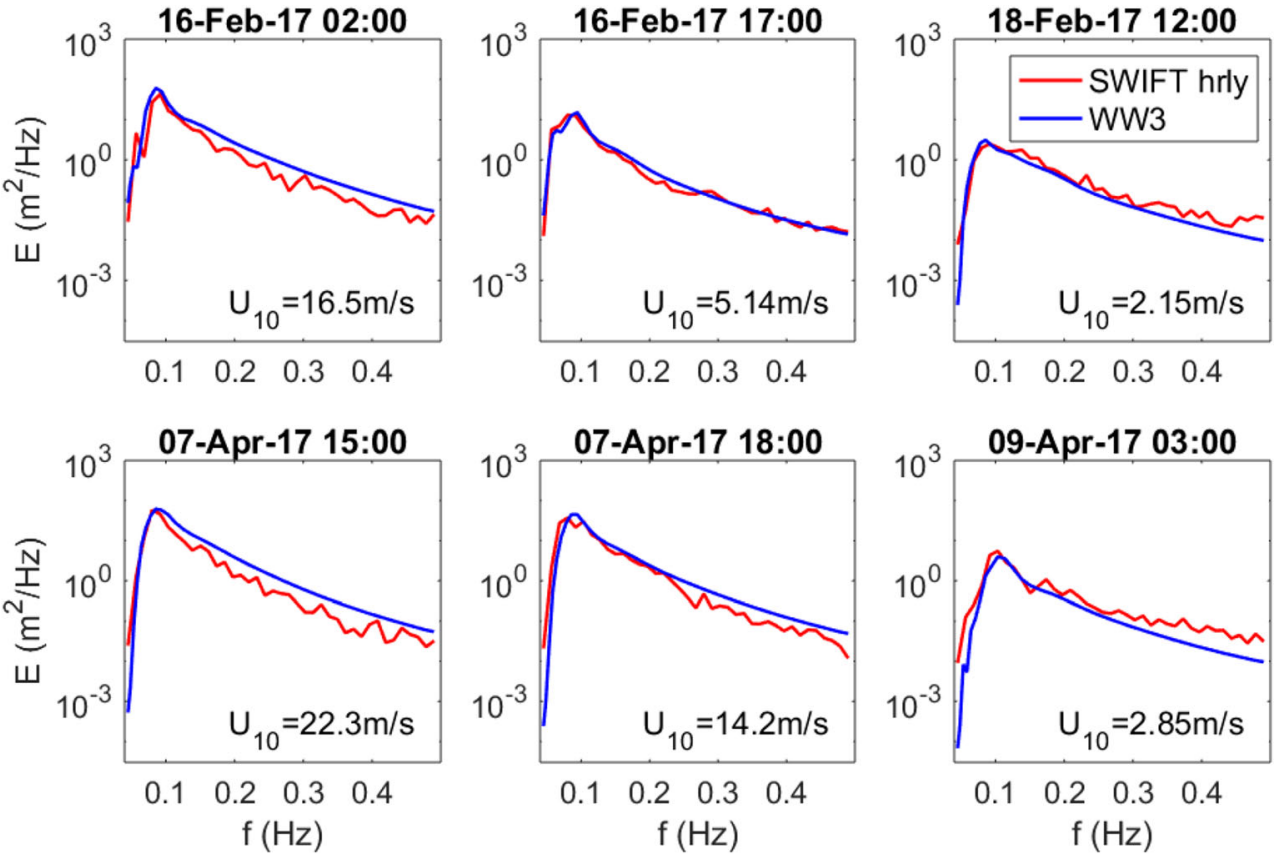

For a closer examination of the wind speed to mean square slope relation, we plot these parameters against each other in Fig. 10. SWIFT mean square slope increases linearly with $U_{10}$ until it levels off at approximately 0.014 for wind speeds greater than $11 \mathrm{~m} / \mathrm{s}$. On the other hand, mss values calculated using WW3 data have an increasing, nearly linear, trend relative to all wind speeds observed. The capping of SWIFTobserved mean squared slope is qualitatively similar to the saturation of SWIFT-observed turbulent dissipation. Likewise, although exponentially distinct, WW3 mean square slope and WW3 bulk dissipation both have an upward trend relative to wind speed.

To ensure these mss findings are not an artifact of our data or specific to the hydrodynamic response of the SWIFT buoy itself, we briefly reference a dataset of wind and wave measurements in the Pacific Northwest presented in Schwendeman and Thomson (2015) and in Thomson et al. (2016). Data acquisition took place in January of 2015 during a voyage aboard the R/V Thomas $G$. Thompson near Ocean Weather Station P (OWS-P, located at $\left.50^{\circ} \mathrm{N}, 145^{\circ} \mathrm{W}\right)$. Wind measurements were collected with a three-axis sonic anemometer mounted on the bow of the ship. Wave spectral data were obtained simultaneously using free-drifting SWIFTs and a moored 0.9-m diameter Datawell directional Waverider MKIII (WR). These data are utilized here to analyze the relationship between mss and wind speed for this independent data set.

Figure 11 shows the mean square slope, derived separately from both SWIFT and Waverider spectra, plotted against wind speed. The average points shown are for SWIFT bins with 30 raw data points (except for the last bin which only has 20), and for Waverider bins with 10 raw data points (except for the last one which only has 9). As with the 2017 dataset, mean SWIFT mss levelsoff at about 0.014 above $U_{10} \approx 11 \mathrm{~m} / \mathrm{s}$, while WR mss caps at about 0.018 above $U_{10} \approx 14 \mathrm{~m} / \mathrm{s}$.
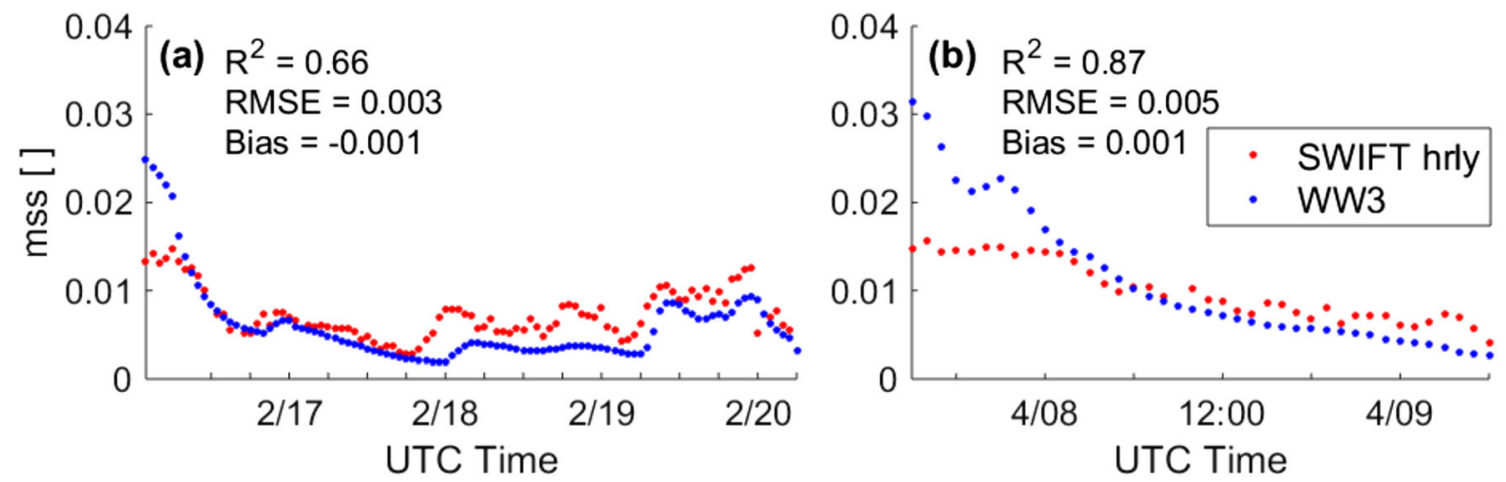

Fig. 9 Time series of mean squared slope for the a February 2017 and b April 2017 deployments. SWIFT mss is shown in red and WW3 mss is shown in blue 
Fig. 10 Mean square slope calculated from SWIFT wave spectra (top) and WW3 wave spectra (bottom) versus 10-m wind speed. Data points corresponding to the February 2017 deployment are shown in cyan and in orange for the April 2017 deployment. Filled circles indicate data points taken at a water depth greater than $50 \mathrm{~m}$. The dashed black line in the top panel is a guideline for the average saturation value of $\mathrm{mss}=$ 0.014 . The solid blue line in the bottom panel is the linear fit to the combined WW3 data points at a water depth greater than $50 \mathrm{~m}$
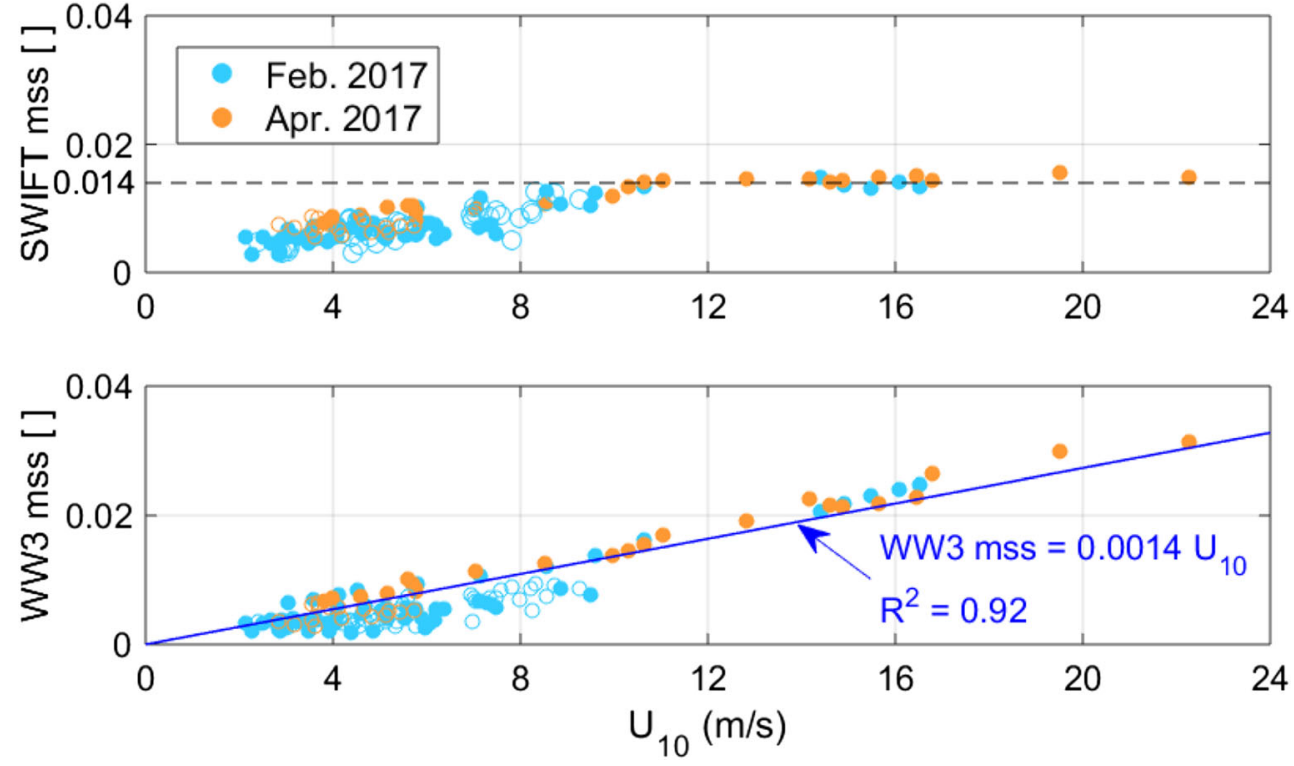

The corroboration of the saturation of mss from the Waverider measurements suggests there are real physical mechanisms at work limiting wave growth with additional wind input. This in turn may limit the amount of wavebreaking dissipation due to turbulence at high winds, even though total wave-breaking dissipation may still increase with increasing wind speed. For example, it is possible that the "clipping" of wave crests and the generation of sea spray, spindrift, and spume at high winds are sufficient mechanisms to prevent waves from increasing steepness beyond the suggested limiting range (mss $\approx 0.014-0.018)$. Such mechanisms would dissipate energy above, or right at, the sea surface, and this dissipation would not be captured in the SWIFT turbulence measurements just below the surface (hence the saturation in those values as well). In this mechanism, the wind input and wave dissipation could both continue to increase with wind speed, but it would not manifest as steeper waves or as more turbulence below the surface. Alternatively, increasing total wave-breaking dissipation in the presence of a limiting turbulent energy dissipation may point towards breaking events that are associated with deeper penetration of bubble clouds (which would also not be captured by the SWIFT turbulence measurements).

\subsection{Consideration of individual breakers}

Breaking waves were identified via a new method introduced in Brown et al. (2018) using the same SWIFT data as in this study. The algorithm involves computing the Short-Time Fourier Transform (STFT) of acceleration time series to highlight rapid magnitude changes as a sign of breaking. Acceleration was resolved in a North-West$\mathrm{Up}$ (NWU) reference frame at a $25-\mathrm{Hz}$ resolution, and the resulting time series were broken into smaller sections of

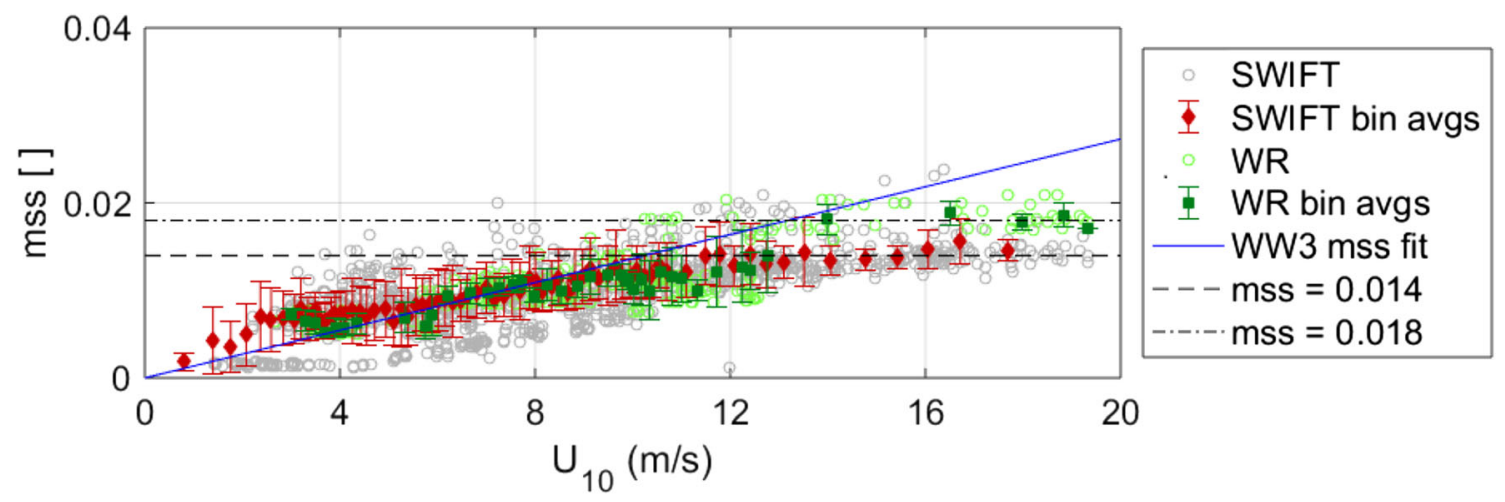

Fig. 11 Measurements of mean square slope versus wind speed at $10 \mathrm{~m}$ elevation obtained using SWIFTs (gray circles) and a Waverider (green circles) at OWS-P. Bin averages are shown in red diamonds for SWIFTs (with 30 elements per bin) and green squares for the Waverider (with 10 elements per bin), with error bars spanning one standard deviation above and below. The solid blue line is the linear fit of $\operatorname{mss}\left(U_{10}\right)$ to WW3 data from Fig. 10. The black dashed and dashed-dotted horizontal lines are guidelines set at $\mathrm{mss}=0.014$ and $\mathrm{mss}=0.018$, respectively 
32 samples (or approximately $1.5 \mathrm{~s}$ ) each tapered with a Blackman window. The Fast Fourier Transform of each short-time window was then computed and the breaking metric $\alpha\left(\mathrm{m} / \mathrm{s}^{2}\right)$ was defined as:

$\alpha=\sum_{f \geq 2}\left|a_{\mathrm{N}}(f)\right|+\sum_{f \geq 2}\left|a_{\mathrm{W}}(f)\right|+\sum_{f \geq 2}\left|a_{\mathrm{U}}(f)\right|$

In Eq. (8), $\left|a_{i}\right|$ is the complex amplitude of the STFT at frequencies $f \geq 2 \mathrm{~Hz}$, and the subscript $i$ represents each direction of the NWU reference frame. Frequencies lower than $2 \mathrm{~Hz}$ were filtered out to limit the influence of the SWIFT's resonant response. For this dataset, waves are said to be breaking during windows with $\alpha$ values greater than $3.25 \mathrm{~m} / \mathrm{s}^{2}$. This empirical threshold was chosen such that for times the algorithm indicated breakers, the corresponding available images confirmed whitecapping.

After identifying breaking waves from the acceleration time series, the breaking probability can be determined by calculating breaker fraction:

$Q_{\mathrm{b}}=N_{\mathrm{b}} T / \tau$

where $N_{\mathrm{b}}$ is the number of observed breakers during a period of observation $\tau$, and $T$ is the corresponding wave period. In Brown et al. (2018), values of $Q_{\mathrm{b}}$ were computed for each data burst ( $\tau=12 \mathrm{~min}$ ). In this study, breaker fraction bursts were averaged over each hour of each deployment.

Breaking waves were also identified visually from video imaging captured by the SWIFT camera at a rate of 635 frames per hour. To reduce the size of the datasets, images captured from sunset to sunrise, and those taken once the buoy reached the coastline, were removed. Images were then categorized based on a three-level criterion in which waves could be breaking, possibly breaking, or not breaking. Waves were classified as breaking when they were photographed just as their crests were breaking near the buoy. Images were also flagged as breaking when the presence of a whitecap indicated a recently broken wave. The images shown in Fig. 3 are examples of breaking waves. Possibly breaking waves were generally those where the presence of glare in the image, or the long-lasting prevalence of drifting foam over seemingly calm water, made it difficult to determine if a wave was breaking or had recently broken. Waves that were thought to be breaking at a distance, but it was uncertain if their impact would have been recorded by the other SWIFT instrumentation, were also flagged as possibly breaking. All remaining images were identified as not breaking.

The total count of possibly breaking and breaking waves identified per hour during the February and April deployments, as well as corresponding breaker fraction estimates, are shown in Fig. 12 and Fig. 13, respectively. In February of 2017 , the $Q_{\mathrm{b}}$ estimates indicate the greatest breaking activity at the beginning of the deployment. Unfortunately, most of the images captured during that time were taken after dark. After that initial stage, most of the images flagged were of possibly breaking waves with few confirmed breakers. Qualitatively, the time series of image counts and breaker fraction are not very well correlated in the February 2017 deployment. By contrast, there is generally good agreement between high values of breaker fraction and high counts of breaking and possibly breaking waves during the April 2017 deployment; as well as low values of breaker fraction when little or no breakers were visually identified. Times when there are discrepancies
Fig. 12 Hourly count of images showing possibly breaking and breaking waves (green and blue bars corresponding, left ordinate) and breaker fraction (orange circles, right ordinate) for the February 2017 deployment. Shading indicates bins in which no images were reviewed
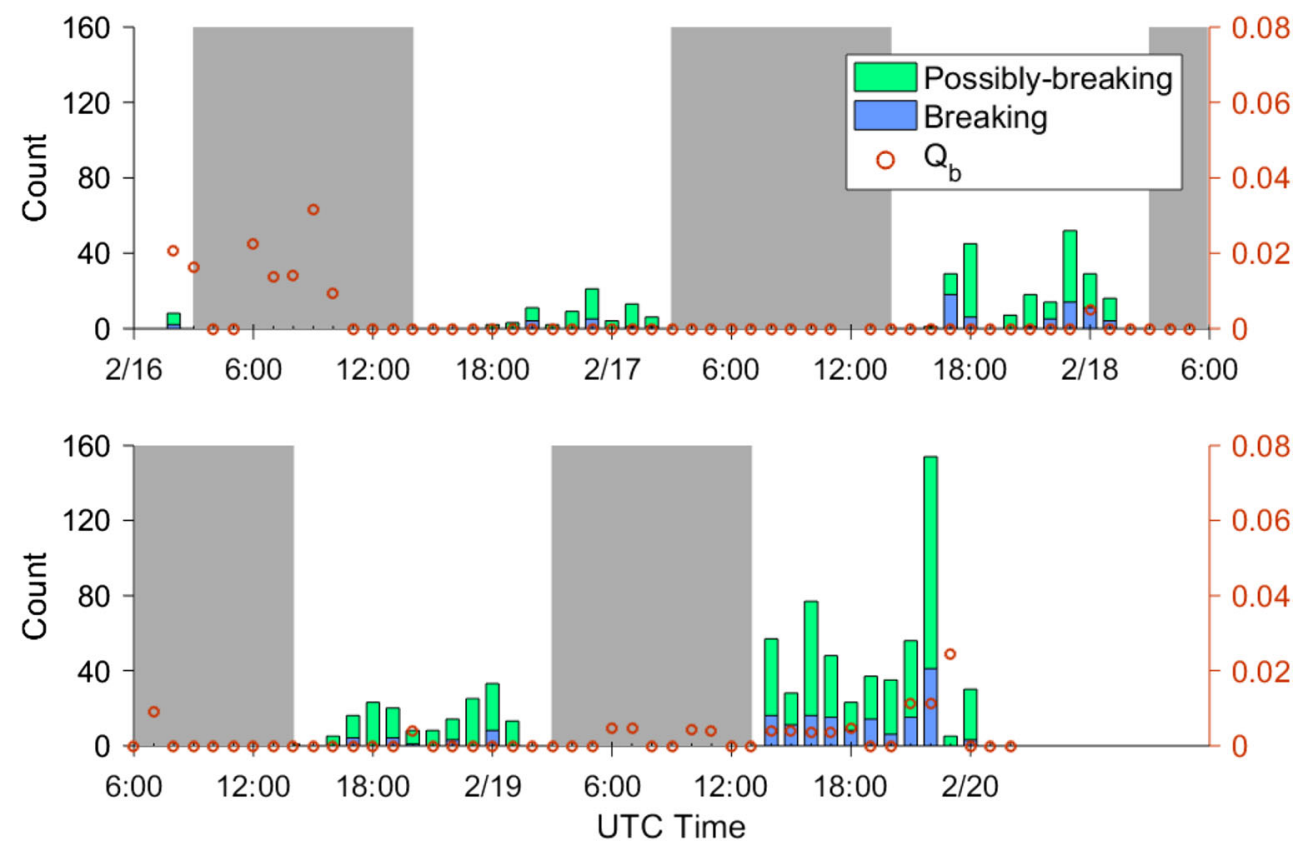
Fig. 13 Hourly count of images showing possibly breaking and breaking waves (green and blue bars corresponding, left ordinate) and breaker fraction (orange circles, right ordinate) for the April 2017 deployment. Shading indicates bins in which no images were reviewed

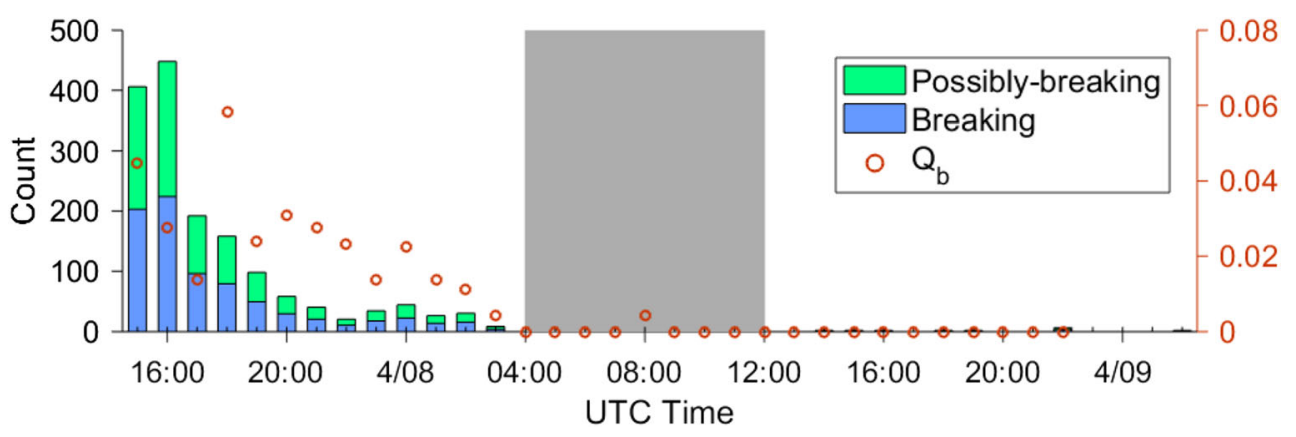

between breaking image counts and breaker fraction may be due to the limitations of the camera footage. True breakers identified as possibly breaking, or perhaps missed altogether because the camera was facing the wrong way, may make breaker fraction values appear too high. Meanwhile, false breakers identified as possibly breaking may make breaker fraction values appear too low. It is also inherently the case, because of instrumentation size limitations, that sufficiently small breaking waves fail to excite the SWIFT acceleration enough to indicate breaking in the Brown et al. (2018) algorithm. Furthermore, in the selection of the breaking threshold obtained from Brown et al. (2018), only the images corresponding to indicated breakers were checked, and not the entire image dataset as was done for this study. As such, it is possible that the resulting breaker fraction values may have been underestimated in the case of small whitecaps, which are reasonably expected to occur during low wind conditions and growing seas.

The relation between breaker fraction and wind speed is shown in Fig. 14. Due to the dependence of breaker fraction on localized acceleration measurements, we bin average $Q_{\mathrm{b}}$ by wind speed (with 8 data points per bin). At wind speeds below $8 \mathrm{~m} / \mathrm{s}$, breaker fraction is small $(\leq 0.01)$ and increases nearly linearly with stronger winds.

A breaking parameter that has been better correlated with wind speed is whitecap coverage; it is most commonly estimated from video imagery and used to approximate wavebreaking dissipation. We are unable to calculate whitecap coverage because of the limited field of view for these images, as well as the inherent complexity in normalizing the camera orientation in each frame. It is worth mentioning, however, that some studies that have delved into determining the best fit to the wind speed-whitecap coverage relationship (typically a power law or cubic function) have observed a leveling off in whitecap coverage with high winds (e.g., Callaghan et al. 2008; Schwendeman and Thomson 2015.). The latest WW3 user manual (The WAVEWATCH III Development Group 2016, corresponding to version 5.16), indicates that whitecap coverage will be made available as a gridded output in a future update. Care should be taken in the model characterization of this parameter since, based on this information, whitecap coverage may be overly estimated at high wind speeds by a power law or cubic fit.

\subsection{Fidelity of wave parameters relative to dissipation}

Here, we assess the significance of dissipation rate on the estimation of bulk parameters, specifically significant wave height, wave period, and wave direction. Regression plots of measured versus modeled bulk parameters, colored by the difference between WW3 total wave-breaking dissipation and SWIFT-observed turbulent dissipation, are shown in Fig. 15. The regressions and accompanying $R$-squared values shown in the figure were estimated for the combined February and April 2017 observations made at water depths greater than $50 \mathrm{~m}$. In panel (a), we see that although significant wave height was estimated quite well using the model $\left(R^{2}=0.95\right)$, there is noticeably more scatter when the difference between

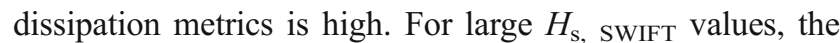
corresponding modeled values are slightly greater, suggesting

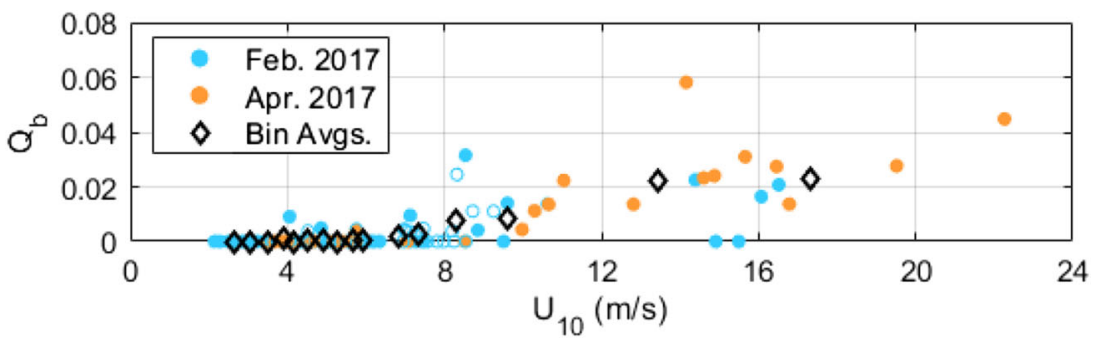

Fig. 14 Breaker fraction versus 10-m wind speed. Data points corresponding to the February and April 2017 deployments are shown in cyan and in orange, respectively; bin averages for the combined data are shown in black (with 8 elements per bin). Filled circles indicate data points taken at a water depth greater than $50 \mathrm{~m}$ 

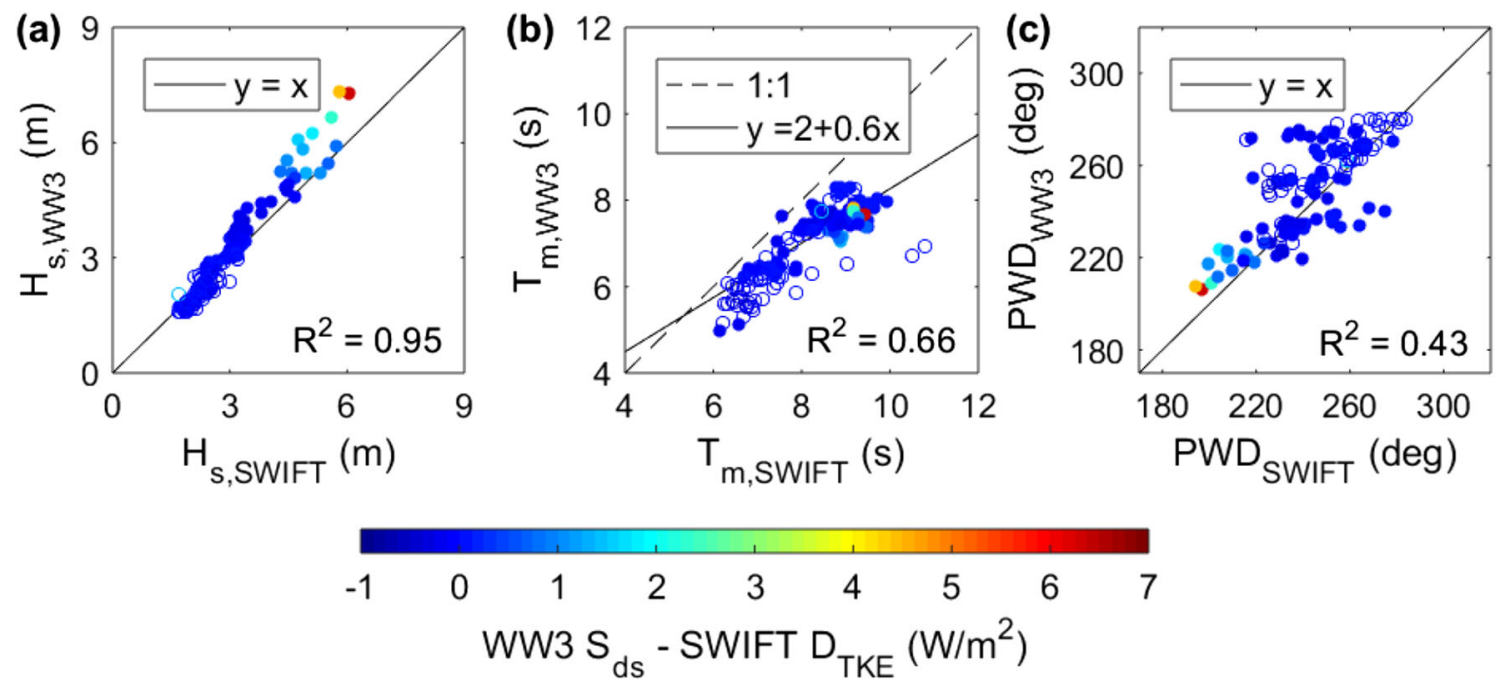

Fig. 15 Linear regressions of SWIFT and WW3 a significant wave height, $\mathbf{b}$ mean wave period, and $\mathbf{c}$ peak wave direction colored by the difference between WW3 wave dissipation and SWIFT turbulent dissipation. The regression and skill $\left(R^{2}\right)$ shown in each panel was calculated only with data points taken at a water depth greater than $50 \mathrm{~m}$ (filled circles) that not enough energy is being dissipated in the model to match the lower significant wave heights observed. The indication that WW3 $S_{\mathrm{ds}}$ may need to be greater to optimize $H_{\mathrm{s}}$ estimates (perhaps making the difference between these dissipation metrics also greater) could seem surprising given that WW $3 S_{\mathrm{ds}}$ is already considerably greater than SWIFT $D_{\mathrm{TKE}}$, but this only highlights the premise that TKE dissipation is only taken as a proxy of total wave dissipation $S_{\mathrm{ds}}$ and is in fact expected to be a smaller fraction of total dissipation when other processes such as bubble production become important. What this panel mainly suggests is that large differences between TKE and total dissipation are associated with a lower skill in the model to estimate significant wave height. In panel (b), we notice once again that mean period is generally underpredicted by the model, yet the errors of estimation do not seem to be directly linked to dissipation. Lastly, the skill of peak wave direction estimates is $R^{2}=0.43$. Although some color groupings in panel (c) hint that larger differences in dissipation metrics are associated with better predictions of PWD, there is too much scatter in the plots to assert this with confidence.

\section{Conclusions}

We describe highly energetic sea states, driven by winds up to $23 \mathrm{~m} / \mathrm{s}$, during two SWIFT deployments made in the winter of 2017. Field measurements of waves and wave breaking collected with the SWIFT are compared with coincident model results from WAVEWATCH III simulations using the ST6 physics package. ST6 wave dissipation is dependent on wave steepness so that the model is internally consistent in continuing to increase both steepness and dissipation with increasing winds.

WW3 estimates of significant wave height are found to have high skill, while the mean wave period is generally underpredicted by the model. Estimates of peak wave direction have moderate skill for the first deployment but a high skill for the second. The unidirectional wave energy spectra are generally well predicted by the model, particularly when wind conditions were moderate.

A direct comparison of turbulent and bulk dissipation rates shows that measured $D_{\mathrm{TKE}}$ only amounts to a fraction of modeled $S_{\mathrm{ds}}$ in the presence of high winds. Occasionally, the opposite is observed, but to a lesser degree; at such times, the model may not be dissipating enough energy. It is worth remembering at this point that turbulent dissipation is only used as a proxy of total wave dissipation and that differences between the two do not necessarily invalidate either. A comparison of these two parameters to wind speed reveals a magnitude saturation of $D_{\mathrm{TKE}}$ at high wind speeds, in contrast to an increasing trend, best fit by a cubic function, for $S_{\mathrm{ds}}$. Similar trends in the relation between mean squared slope and wind speed are found - this time with modeled mss having a more linear relation with wind speed while measured mss saturates at a value of 0.014 for strong winds. The saturation of mean squared slope observed by the SWIFT is consistent with similar measurements collected with a Datawell Waverider buoy near Ocean Weather Station P, with mss measurements saturating at about 0.018 .

The saturation in the measured turbulence and mean square slopes, in combination with increasing total dissipation in the wavefield with increasing wind, suggests the existence of a different dynamic regime for wave breaking for winds above 
$15 \mathrm{~m} / \mathrm{s}$, where dissipation due to processes such as spindrift or bubble clouds become increasingly important and contribute to the increasing dissipation with increasing wind speed despite saturation in turbulence. A better understanding of the relative importance of the various whitecapping dissipation mechanisms is crucial before attempting to improve the estimation of total deep-water energy dissipation in WW3. Further direct observations of these processes are required as well as additional studies that involve higher resolution modeling. The saturation of turbulence and mean squared slope should also be explored at greater length not only as they pertain to moderately large wind events but also considering swell conditions.

Video imaging data confirms that waves were breaking particularly at the beginning of each deployment. For a more qualitative analysis of whitecaps, we obtained breaker fraction estimates from a companion publication: Brown et al. (2018). The time series of breaker fraction estimates were found to correlate well with the image counts of breakers and possible-breakers identified visually, but more so for the April 2017 deployment. On average, an increasing linear trend is observed between breaker fraction and wind speed, in contrast to the saturation of whitecap coverage reported in other studies.

The skill of the model in simulating the encountered field conditions was further analyzed in the context of energy dissipation. When compared with SWIFT measures, significant wave height has lower skill when the difference between modeled $S_{\mathrm{ds}}$ and measured $D_{\mathrm{TKE}}$ is high. No conclusive arguments could be made about the accuracy of wave period and direction estimates relative to the difference of dissipation metrics.

Acknowledgments Alex de Klerk and Joe Talbert prepared the SWIFT for deployment. Brimm Aviation carried out the helicopter deployments.

Funding information We are thankful to the US Department of Energy whose support funds the Advanced Laboratory and Field Arrays (ALFA) for Marine Energy project (task 3).

Open Access This article is distributed under the terms of the Creative Commons Attribution 4.0 International License (http:// creativecommons.org/licenses/by/4.0/), which permits unrestricted use, distribution, and reproduction in any medium, provided you give appropriate credit to the original author(s) and the source, provide a link to the Creative Commons license, and indicate if changes were made.

\section{References}

Amante, C., \& Eakins, B.W. (2009). ETOPO1 1 arc-minute global relief model: procedures, data sources and analysis. NOAA technical memorandum NESDIS NGDC-24. Natl Geophys Data Cent, NOAA. https://doi.org/10.7289/V5C8276M
Ardhuin F, Rogers WE, Babanin AV, Filipot J, Magne R, Roland A, van der Westhuysen A, Queffeulou P, Lefevre J, Aouf L, Collard F (2010) Semiempirical dissipation source functions for ocean waves. Part I: definition, calibration, and validation. J Phys Oceanogr 40(9): 1917-1941. https://doi.org/10.1175/2010JPO4324.1

Banner M, Gemmrich J, Farmer D (2002) Multiscale measurements of ocean wave breaking probability. J Phys Oceanogr 32(12):33643375. https://doi.org/10.1175/1520-0485(2002)032<3364: MMOOWB $>2.0 . \mathrm{CO} ; 2$

Bi F, Song J, Wu K, Xu Y (2015) Evaluation of the simulation capability of the Wavewatch III model for Pacific Ocean wave. Acta Oceanol Sin 34(9):43-57. https://doi.org/10.1007/s13131-015-0737-1

Blenkinsopp C, Chaplin J (2007) Void fraction measurements in breaking waves. Proceedings: Mathematical Phys and Eng Sci 463(2088): 3151-3170. https://doi.org/10.1098/rspa.2007.1901

Brown A, Thomson J, Ellenson A, Ticona Rollano F, Özkan-Haller HT, Haller MC (2018) Kinematics and statistics of breaking waves observed using SWIFT buoys. J Ocean Eng. https://doi.org/10.1109/ JOE.2018.2868335

Callaghan A, De Leeuw G, Cohen L, O'Dowd C (2008) Relationship of oceanic whitecap coverage to wind speed and wind history. Geophys Res Lett 35:L23609. https://doi.org/10.1029/ 2008GL036165

Carini R, Chickadel C, Jessup A, Thomson J (2015) Estimating wave energy dissipation in the surf zone using thermal infrared imagery. $\mathrm{J}$ Geophys Res: Oceans 120(6):3937-3957. https://doi.org/10.1002/ 2014JC010561

Craig P, Banner M (1994) Modeling wave-enhanced turbulence in the ocean surface layer. J Phys Oceanogr 24(12):2546-2559. https://doi. org/10.1175/1520-0485(1994)024<2546:MWETIT>2.0.CO;2

Deane G, Stokes M, Callaghan A (2016) The saturation of fluid turbulence in breaking laboratory waves and implications for whitecaps. J Phys Oceanogr 46(3):975-992. https://doi.org/10.1175/JPO-D-140187.1

Deike L, Melville W, Popinet S (2016) Air entrainment and bubble statistics in breaking waves. J Fluid Mech 801:91-129. https://doi.org/ 10.1017/jfm.2016.372

Donelan M, Babanin A, Young I, Banner M (2006) Wave-follower field measurements of the wind-input spectral function. Part II: parameterization of the wind input. J Phys Oceanogr 36(8):1672-1689. https://doi.org/10.1175/JPO2933.1

Ellenson A, Özkan-Haller HT (2018) Predicting large ocean wave events characterized by bimodal energy spectra in the presence of a lowlevel southerly wind feature. Weather and Forecast 33(2):479-499. https://doi.org/10.1175/waf-d-17-0035.1

García-Medina G, Özkan-Haller HT, Ruggiero P (2014) Wave resource assessment in Oregon and southwest Washington, USA. Renew Energy 64:203-214. https://doi.org/10.1016/j.renene.2013.11.014

Gemmrich J (2010) Strong turbulence in the wave crest region. J Phys Oceanogr 40(3):583-595. https://doi.org/10.1175/2009JPO4179.1

Hasselmann S, Hasselmann K, Allender JH, Barnett P, T.P. (1985) Computations and parameterizations of the nonlinear energy transfer in a gravity-wave spectrum, Pt. II, parameterizations of the nonlinear energy transfer for application in wave models. J Phys Oceanogr 15(11):1378-1391. https://doi.org/10.1175/1520-0485(1985) 015<1378:CAPOTN $>2.0 . \mathrm{CO} ; 2$

Lamarre E, Melville WK (1991) Air entrainment and dissipation in breaking waves. Nat 351(6326):469-472. https://doi.org/10.1038/ $351469 \mathrm{a} 0$

Melville W (1996) The role of surface-wave breaking in Air-Sea interaction. Annu Rev Fluid Mech 28(1):279-321. https://doi.org/10.1146/ annurev.fl.28.010196.001431

Phillips O (1985) Spectral and statistical properties of the equilibrium range in wind-generated gravity waves. J Fluid Mech 156:505531. https://doi.org/10.1017/S0022112085002221 
Schwendeman M, Thomson J, Gemmrich J (2014) Wave breaking dissipation in a Young Wind Sea. J Phys Oceanogr 44(1):104-127. https://doi.org/10.1175/JPO-D-12-0237.1

Schwendeman M, Thomson J (2015) Observations of whitecap coverage and the relation to wind stress, wave slope, and turbulent dissipation. J Geophys Res: Oceans 120(12):8346-8363. https://doi.org/10. 1002/2015JC011196

Sutherland P, Melville W (2015) Field measurements of surface and nearsurface turbulence in the presence of breaking waves. J Phys Oceanogr 45(4):943-965. https://doi.org/10.1175/JPO-D-14-0133.1

Terray E, Donelan M, Agrawal Y, Drennan W, Kahma K, Williams A, Hwang P, Kitaigorodskii S (1996) Estimates of kinetic energy dissipation under breaking waves. J Phys Oceanogr 26(5):792-807. https://doi.org/10.1175/1520-0485(1996)026<0792:EOKEDU>2.0. $\mathrm{CO} ; 2$

Thomson J (2012) Wave breaking dissipation observed with "SWIFT" drifters. J Atmos Ocean Technol 29(12):1866-1882. https://doi.org/ 10.1175/jtech-d-12-00018.1

Thomson J, Schwendeman MS, Zippel SF, Moghimi S, Gemmrich J, Rogers WE (2016) Wave-breaking turbulence in the ocean surface layer. J Phys Oceanogr 46(6):1857-1870. https://doi.org/10.1175/ jpo-d-15-0130.1

The Wavewatch III Development Group (2016). User manual and system documentation of WAVEWATCH III version 5.16. Tech. Note 329, NOAA/NWS/NCEP/MMAB, College Park, MD, USA, 326 pp. + Appendices

Tolman H, Chalikov D (1996) Source terms in a third-generation wind wave model. J Phys Oceanogr 26(11):2497-2524. https://doi.org/ 10.1175/1520-0485(1996)026<2497:STIATG >2.0.CO;2

Yang Z, Neary V (2017) A wave model test bed study for wave energy resource characterization. Renew Energy 114(PA):132-144. https:// doi.org/10.1016/j.renene.2016.12.057

Zieger S, Babanin AV, Rogers WE, Young IR (2015) Observation-based source terms in the third-generation wave model WAVEWATCH. Ocean Model 96:2-25. https://doi.org/10.1016/j.ocemod.2015.07.014

Zippel S, Thomson J (2015) Wave breaking and turbulence at a tidal inlet. J Geophys Res: Oceans 120(2):1016-1031. https://doi.org/10.1002/ 2014jc010025 